\title{
Space-occupying cerebellar infarction: complications, treatment, and outcome
}

\author{
Hermann Neugebauer, M.D., M.Sc., ${ }^{1}$ Jens Witsch, M.D., ${ }^{2}$ Klaus Zweckberger, M.D., ${ }^{3}$ \\ ANd Eric Jüttler, M.D., M.Sc. ${ }^{1}$ \\ ${ }^{1}$ Department of Neurology, Rehabilitation and University Hospital Ulm; ${ }^{2}$ Department of Neurology, Charité \\ University Medicine Berlin, Campus Virchow Klinikum, Berlin; and ${ }^{3}$ Department of Neurosurgery, \\ Ruprecht-Karl-University Heidelberg, Germany
}

\begin{abstract}
Space-occupying brain edema is a frequent and one of the most dreaded complications in ischemic cerebellar stroke. Because the tight posterior fossa provides little compensating space, any space-occupying lesion can lead to life-threatening complications through brainstem compression or compression of the fourth ventricle and subsequent hydrocephalus, both of which may portend transtentorial/transforaminal herniation. Patients with large cerebellar infarcts should be treated and monitored very early on in an experienced stroke unit or (neuro)intensive care unit. The general treatment of ischemic cerebellar infarction does not differ from that of supratentorial ischemic strokes. Treatment strategies for space-occupying edema include pharmacological antiedema and intracranial pressure-lowering therapies, ventricular drainage by means of an extraventricular drain, and suboccipital decompressive surgery, with or without resection of necrotic tissue. Timely escalation of treatment is crucial and should be guided by clinical and neuroradiological rationales. Patients in a coma after hydrocephalus and/or local brainstem compression may also benefit from more aggressive surgical treatment, as long as the conditions are reversible. Contrary to the general belief that outcome in survivors of space-occupying cerebellar stroke is usually good, recent studies suggest that for many of these patients, the long-term outcome is not good. In particular, advanced age and additional brainstem infarction seem to be predictors for poor outcome. Further trials are necessary to investigate these findings systematically and provide better selection criteria to help guide decisions about surgical therapies, which should always be carried out in close cooperation among neurointensive care physicians, neurologists, and neurosurgeons.
\end{abstract} (http://thejns.org/doi/abs/10.3171/2013.2.FOCUS12363)

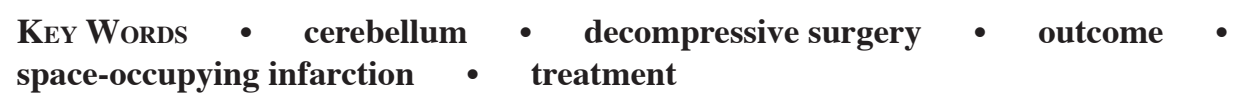

$\mathrm{T}$ He first case reports involving patients with spaceoccupying cerebellar infarcts date back to the end of the 19 th century ${ }^{80}$ The first case reports of surgical treatment by craniectomy were published independently but almost simultaneously in 1956 by Fairburn and Olivier ${ }^{26}$ and Lindgren. ${ }^{73}$ As of the writing of this study, 115 original papers on space-occupying cerebellar stroke including a total of over 750 patients have been published (see Table 3).

The exact number of patients who develop space-occupying edema after cerebellar infarction is not known. Data from the literature show heterogeneous results,

\footnotetext{
Abbreviations used in this paper: $\mathrm{BAEP}=$ brainstem auditory evoked potentials; $\mathrm{BI}=$ Barthel Index $; \mathrm{EVD}=$ external ventricular drainage; GASCIS = the German-Austrian Space-Occupying Cerebellar Infarction Study; GOS = Glasgow Outcome Scale; ICP = intracranial pressure; $\mathrm{mRS}=$ modified Rankin Scale; $\mathrm{SDC}=$ suboccipital decompressive craniectomy; SEP = somatosensory evoked potentials.
}

ranging from $17 \%$ to $54 \% .15,19,41,47,51,58,75,84,94,104,112$ The proportion of patients in which infarct swelling is potentially life-threatening also remains uncertain. In one study only $50 \%$ of patients who developed radiographic evidence of mass effect showed subsequent clinical deterioration. ${ }^{63}$

The diagnosis and management of cerebellar stroke with mass effect is still a controversial issue. Because of nonspecific symptoms ${ }^{47,51,58,75,84,112}$ and insufficient diagnostic sensitivity of brain CT (the most widely available and commonly used imaging technique in the evaluation of patients with suspected stroke) ${ }^{77}$ large cerebellar infarctions prone to space-occupying edema formation are often not recognized until complications due to mass effect become evident with clinical deterioration. The most feared complications in the posterior fossa are brainstem compression and occlusive hydrocephalus. In the case of extensive mass effect, conservative treatment strategies are not well validated and are often unsuccessful in clinical practice; surgical treatment is widely accepted 


\section{H. Neugebauer et al.}

as the treatment of choice. However, the best time point for starting and the factors triggering surgical intervention remain unclear. Furthermore, the different surgical procedures have not been sufficiently tested in larger, multicenter, prospective, or randomized controlled trials. Therefore, management strategies are often based solely on personal experience or local standards and differ between neurological/neurosurgical centers. Although numerous reports suggest that surgical intervention reduces mortality in malignant cerebellar infarction, little is known about the long-term outcome in survivors.

The current work provides an overview of the available data on complications, treatment, and outcome of space-occupying cerebellar infarction.

\section{Methods}

References for this review were identified by searches of PubMed, Medline and local search machines in the library of the Charité-Berlin up to October 2012 using the terms "cerebellar," "stroke," "ischaemia," "infarction," "malignant," "space-occupying," "swelling," "extraventricular," "drainage," "decompressive," "surgery," "surgical," "decompression," "craniectomy," and "suboccipital" in various combinations. Articles were also identified through searches of the authors' own files. Papers published in English or German and papers providing an abstract in either language were reviewed. All articles reporting outcome data on space-occupying cerebellar infarction were considered for the analysis. Primary data of studies that enrolled a large number of patients were given priority over case reports and reviews with respect to treatment recommendations.

\section{Complications}

Large infarcts of the cerebellar hemispheres, particularly those affecting the posterior inferior cerebellar artery and infarcts affecting multiple arterial territories may be accompanied by the development of brain swelling due to cytotoxic and vasogenic edema. ${ }^{15,19,41,47,51,56,58,75,84,91,94,104}$ So far it is not clear which factors promote edema formation after stroke. In addition to infarct size, other factors such as hemorrhagic transformation and inadequate collateral blood flow may be involved. Interestingly, large infarcts can be accompanied by only a small amount of edema, whereas comparatively small infarcts can result in extensive swelling. ${ }^{63}$ The timing of edema formation can vary. According to data from neuroimaging studies, maximum swelling is reached in most patients between the 2 nd and the 4 th day but has also been reported as late as the 9th day, when patients were already regarded as being stable..$^{15,47,51,58,61}$

The 2 major complications of edema formation in the posterior fossa are obstructive hydrocephalus due to blockage of the fourth ventricle and direct compression of the midbrain and pons. Upward transtentorial herniation of the superior vermis cerebelli through the tentorial notch or downward herniation of the cerebellar tonsils through the foramen magnum are the most common causes of death in the acute phase. . $^{3,41,51,58,75,94,104,112}$

\section{Treatment}

\section{Conservative Treatment}

Guidelines for conservative treatment are derived from those for acute ischemic stroke in general and include airway protection, adequate oxygenation, blood pressure management, management of blood glucose levels, body temperature, and prevention of deep venous thrombosis and pulmonary embolism. ${ }^{1,25}$ The level of consciousness should be monitored closely. Immediate imaging should be prompted in the case of clinical deterioration or occurrence of brainstem signs. Escalation of conservative treatment includes ventilation, deep sedation, and other intensive care measures. Medical treatment strategies for increased ICP are in accordance with those used in the management of supratentorial stroke and may include osmotic therapy, artificial coma, hyperventilation, barbiturates, buffers, and steroids. ${ }^{8,55}$ However, none of these treatments is supported by adequate evidence of efficacy from experimental studies or randomized clinical trials. ${ }^{1,25}$ In fact, several reports suggest that these measures are ineffective or even detrimental, at least in supratentorial infarction. ${ }^{8,55}$ No valid data are available in infratentorial stroke.

\section{Surgical Treatment}

Surgical therapy includes ventriculostomy and suboccipital decompressive craniectomy (SDC). In contrast to the controversial debate about surgical treatment of space-occupying supratentorial stroke, surgical intervention is widely accepted as the treatment of choice in cerebellar stroke with mass effect and strongly recommended in current stroke guidelines. ${ }^{1,25}$

\section{Ventricular Drainage}

Ventriculostomy is most commonly performed by inserting an extraventricular drain in a lateral ventricle. This procedure represents the gold standard of treatment and offers the opportunity for both measuring the supratentorial ICP and, if necessary, draining CSF. Although few authors have described a lower risk for infections in endoscopic ventriculostomy, this technique remains an individual approach (only used by some surgeons) and entails, besides a higher risk of surgical complications, the additional risk that CSF drainage is not controllable and might potentially be insufficient..$^{7123}$

\section{Suboccipital Craniectomy}

The rational for suboccipital decompressive surgery is to create space for the swollen tissue outside the narrow posterior fossa and thus avoid life-threatening compression of the brainstem and fourth ventricle. There are various surgical approaches: unilateral or bilateral craniectomy, additional opening of the foramen magnum with or without resection of the posterior arch of the atlas, and the use of different types of duraplasty. Some neurosurgeons also remove necrotic cerebellar tissue to create extra space within the posterior fossa and reduce development of cytotoxic edema. ${ }^{51,56,91}$

In cerebellar stroke, there is no general agreement about the optimal time to initiate surgery or about what 
factors would mandate proceeding to surgery. Local surgery protocols differ greatly, and the questions of whether and when ventriculostomy or SDC should be performed and whether surgery should be performed in a single intervention, in combination, or in a stepwise approach remain unanswered. It is important to note that none of these procedures has been tested or compared with others in larger, multicenter, and prospective studies with adequate control groups or in randomized trials.

As for SDC, the following protocol may be used: Patients are operated in a prone or semiprone (park-bench) position. After vertical midline skin incision from the protuberantia occipitalis to the upper cervical spine, skin and subcutaneous tissue are separated from the underlying fascia, which is cut through laterally to the processi spinosi of the upper cervical spine, and muscles are disconnected from the processi spinosi and the occiput. Osteoclastic bilateral craniotomy is performed beneath the transverse sinus and enlarged laterally. Great attention must be paid to opening the foramen magnum sufficiently to decompress the cerebellar tonsils. Resection of the posterior arch of the atlas is recommended in order to achieve sufficient decompression. To maximize the decompressive effect, the dura should be opened (Y-shaped) above the cerebellar hemispheres and the medulla oblongata. If the effect of decompression seems insufficient, removal of necrotic tissue might be considered as an additional option. Finally, the dura should be reconstructed by means of duraplasty with an autologous galea or fascia flap or, more commonly, an artificial dural substitute. Complications such as injury to the vertebral arteries, induction of major bleeding, or epidural and subdural hematoma and hygroma should be avoided. ${ }^{118}$

\section{Outcome}

The analysis of available studies on space-occupying cerebellar stroke showed that conservative treatment alone is associated with a high mortality rate of $42.9 \%$, increasing to a rate as high as $85 \%$ in those patients who progress to coma..$^{27,41}$

Surgical treatment is associated with much higher survival rates: $81.6 \%$ for patients treated with EVD alone, $76.8 \%$ for those treated with SDC alone, and $77.5 \%$ for those treated with both EVD and SDC. Mortality and functional outcomes are summarized in Tables 1 and 2 . There is some evidence that higher age is associated with poorer outcome. .7,56 $^{4}$

The summary tables should be interpreted with caution as most of the studies investigating outcome suffer from the limitations of retrospective data collection, lack of control groups, single-center or small multicenter (oligocenter) experience, limited size of patient cohorts, and other selection biases (Table 3). Most importantly, there were marked imbalances between treatment groups; i.e., patients with pronounced mass effect on CT and impaired level of consciousness were more likely to be treated with SDC and additional EVD. Most studies do not provide information about factors predicting the course of the disease or functional outcome in survivors.

The only study with a prospective multicenter design
TABLE 1: Mortality stratified by treatment strategy in 764 patients with space-occupying cerebellar stroke*

\begin{tabular}{lrr}
\hline \multicolumn{1}{c}{ Variable } & \multicolumn{1}{c}{ Dead } & Survived \\
\hline overall outcome & $213(27.9)$ & $551(72.1)$ \\
conservative treatment & $85(42.9)$ & $113(57.1)$ \\
SDC w/o EVD & $29(23.2)$ & $96(76.8)$ \\
SDC w/ EVD & $39(22.5)$ & $134(77.5)$ \\
EVD alone & $27(18.4)$ & $120(81.6)$ \\
SDC w/ strokectomy $\dagger$ & $33(26.6)$ & $91(73.4)$ \\
\hline
\end{tabular}

* Values represent numbers of patients (\%).

$\dagger$ Patients in this category are also included in the SDC w/o EVD or $\mathrm{SDC}$ w/ EVD categories above.

addressing clinical outcome after space-occupying cerebellar stroke is the German-Austrian Space-Occupying Cerebellar Infarction Study (GASCIS). ${ }^{51}$ In GASCIS, the level of consciousness before treatment was found to be the only predictor for poor outcome. Patients who were awake/drowsy or somnolent/stuporous had a good functional outcome (mRS score $0-2$ ) in $86 \%$ and $76 \%$ of cases, respectively, while only $47 \%$ of comatose patients had a good outcome. GASCIS was not randomized and therefore was subject to selection bias. In addition, 22.2\% of patients treated with ventriculostomy were later treated with decompressive surgery due to further deterioration.

The observation that about $50 \%$ of patients who progress to coma and are treated with craniectomy have good outcomes (mRS score $\leq 2$ ) is seen in some of the published studies, but others found no association between the initial level of consciousness or its course and functional outcome. $44,47,51,57,68,69,91,112,115$

Today most neurosurgeons and neurologists believe that the clinical outcome of patients with space-occupying cerebellar stroke who have undergone successful life-saving surgery is usually good irrespective of other prognostic factors. This opinion is reflected by current guidelines and recommendations. ${ }^{1,12,25,51,78,94}$ However, little is known about the long-term outcome in survivors. There are only

TABLE 2: Outcome stratified by treatment strategy in 299 survivors of space-occupying cerebellar stroke

\begin{tabular}{lccc}
\hline \multirow{2}{*}{\multicolumn{1}{c}{ Variable }} & \multicolumn{3}{c}{ Disability (\%)* } \\
\cline { 2 - 4 } & None or Mild & Moderate & Severe \\
\hline overall outcome & $162(54.4)$ & $101(33.9)$ & $35(11.7)$ \\
conservative treatment & $58(78.4)$ & $15(20.3)$ & $1(1.4)$ \\
SDC w/o EVD & $13(40.6)$ & $13(40.6)$ & $6(18.8)$ \\
SDC w/ EVD & $39(45.3)$ & $33(38.4)$ & $14(16.3)$ \\
EVD alone & $36(50.7)$ & $25(35.2)$ & $10(14.1)$ \\
SDC w/ strokectomy $\dagger$ & $50(47.6)$ & $43(41.0)$ & $12(11.4)$ \\
\hline
\end{tabular}

* None or mild disability was defined as mRS Score 0 or 1, GOS Score 5, or BI Score 90-100; moderate disability as mRS Score 2 or 3, GOS Score 4, or BI Score 65-85; and severe disability as mRS Score 4 or 5 , GOS Score 2 or 3 , or BI Score $\leq 60$.

$\dagger$ Patients in this category are also included in the SDC w/o EVD or SDC w/ EVD categories above. 


\section{H. Neugebauer et al.}

TABLE 3: Summary of available publications reporting on patients with space-occupying cerebellar infarction*

\begin{tabular}{|c|c|c|c|c|c|c|c|c|}
\hline Authors \& Year & $\begin{array}{l}\text { No. of } \\
\text { Pts† }\end{array}$ & $\begin{array}{c}\text { Mean } \\
\text { Age (yrs) }\end{array}$ & Sex & $\begin{array}{l}\text { Conservative } \\
\text { Treatment }\end{array}$ & SDC w/o EVD & SDC w/ EVD & EVD Alone & $\begin{array}{l}\text { Mean FU in } \\
\text { Mos (range) }\end{array}$ \\
\hline Menzies, 1893 & 1 & 27 & $1 \mathrm{~F}$ & 1 (dead) & 0 & 0 & 0 & NR \\
\hline $\begin{array}{l}\text { Goodhart \& Davidson, } \\
1936\end{array}$ & 1 & 66 & $1 \mathrm{~F}$ & 1 (dead) & 0 & 0 & 0 & NR \\
\hline $\begin{array}{l}\text { Germain \& Morvan, } \\
\quad 1936\end{array}$ & 1 & 32 & $1 \mathrm{M}$ & 1 (dead) & 0 & 0 & 0 & NR \\
\hline Kubik \& Adams, 1946 & 2 & 75 & $1 \mathrm{~F}, 1 \mathrm{M}$ & 2 (dead) & 0 & 0 & 0 & NR \\
\hline Lindgren, 1956 & 2 & 47.5 & $2 \mathrm{M}$ & 0 & $\begin{array}{l}2 \text { (1 dead, } 1 \text { surv, outc } \\
\text { NR) }\end{array}$ & 0 & 0 & 1 \\
\hline $\begin{array}{l}\text { Fairburn \& Oliver, } \\
\quad 1956\end{array}$ & 3 & 58.3 & $3 \mathrm{M}$ & $\begin{array}{l}2 \text { (1 dead, } 1 \\
\text { w/o disab) }\end{array}$ & $1(+)$ (dead) & 0 & 0 & $19(5-33)$ \\
\hline Ford \& Clark, 1956 & 1 & 37 & $1 \mathrm{M}$ & 1 (dead) & 0 & 0 & 0 & NR \\
\hline Pomme et al., 1959 & 2 & 59.5 & $2 \mathrm{M}$ & 0 & $\begin{array}{l}2 \text { (1 dead, } 1 \text { surv, outc } \\
\text { NR) }\end{array}$ & 0 & 0 & NR \\
\hline Fisher et al., 1961 & 2 & 48.5 & $2 \mathrm{~F}$ & 2 (dead) & 0 & 0 & 0 & NR \\
\hline Norris et al., 1969 & 3 & NR & NR & 0 & 3 (3 surv, outc NR) & 0 & 0 & NR \\
\hline $\begin{array}{l}\text { Wood \& Murphey, } \\
1969\end{array}$ & 5 & 43.8 & $5 \mathrm{M}$ & 0 & $\begin{array}{l}5 \text { (2 dead, } 3 \text { surv, outc } \\
\quad \text { NR) }\end{array}$ & 0 & 0 & NR \\
\hline Lehrich et al., 1970 & 4 & 59.3 & $1 \mathrm{~F}, 3 \mathrm{M}$ & 0 & $\begin{array}{l}3(3+) \text { ( } 1 \text { dead, } 2 \text { surv, } \\
\text { outc NR) }\end{array}$ & 0 & 1 (dead) & $43(1-85)$ \\
\hline Cohadon et al., 1970 & 2 & 36 & $1 \mathrm{~F}$ & 0 & 2 (2 surv, outcome NR) & 0 & 0 & NR \\
\hline Corcos et al., 1971 & 1 & NR & NR & 0 & 1 (dead) & 0 & 0 & NR \\
\hline Fischer et al., 1972 & 1 & 0.4 & $1 \mathrm{M}$ & 0 & 0 & 1 (surv, outc NR) & 0 & 4.5 \\
\hline $\begin{array}{l}\text { Graham \& Adams, } \\
\quad 1972\end{array}$ & 2 & 30 & $2 \mathrm{M}$ & 1 (dead) & 0 & 0 & 1 (dead) & NR \\
\hline $\begin{array}{l}\text { Momose \& Lehrich, } \\
1973\end{array}$ & 13 & 52.9 & $\begin{array}{c}6 \mathrm{~F}, 6 \mathrm{M} \\
1 \mathrm{NR}\end{array}$ & 4 (4 dead) & $\begin{array}{l}9(9+)(1 \text { dead, } 8 \text { surv, } \\
\text { outc NR) }\end{array}$ & 0 & 0 & NR \\
\hline Sypert \& Alvord, 1975 & 28 & 63 & $7 \mathrm{~F}, 21 \mathrm{M}$ & 28 (28 dead) & 0 & 0 & 0 & NR \\
\hline Duncan et al., 1975 & 2 & 65.5 & $2 \mathrm{M}$ & 1 (dead) & 1 (surv, mRS0) & 0 & 0 & 2 \\
\hline Ferguson et al., 1976 & 1 & NR & NR & 0 & 0 & 1 (surv, outc NR) & 0 & NR \\
\hline Savitz et al., 1977 & 1 & 60 & $1 \mathrm{M}$ & 0 & 0 & 1 (surv, mRS1) & 0 & 12 \\
\hline Miki et al., 1978 & 2 & NR & NR & 0 & 2 (2 surv, outc NR) & 0 & 0 & NR \\
\hline Levina, 1978 & 1 & NR & NR & NR & NR & NR & NR & NR \\
\hline Géraud et al., 1978 & 3 & NR & NR & 0 & 0 & $\begin{array}{l}2 \text { (1 dead, } 1 \text { surv, } \\
\quad \text { outc NR) }\end{array}$ & $\begin{array}{l}1 \text { (1 surv w/ shunt, } \\
\text { outc NR) }\end{array}$ & NR \\
\hline $\begin{array}{l}\text { Vereshchagin et al., } \\
1978\end{array}$ & 1 & NR & NR & 0 & 1 (surv, outc NR) & 0 & 0 & NR \\
\hline $\begin{array}{l}\text { De Reuck \& Vander } \\
\text { Eecken, } 1978\end{array}$ & 4 & 64.5 & $1 \mathrm{~F}, 3 \mathrm{M}$ & 3 (3 dead) & 0 & 0 & 1 (surv, outc NR) & NR \\
\hline George et al., 1978 & 4 & NR & NR & 0 & $\begin{array}{l}4 \text { (2 dead, } 2 \text { surv, outc } \\
\quad \text { NR) }\end{array}$ & 0 & 0 & NR \\
\hline Arnow et al., 1978 & 1 & 19 & $1 \mathrm{M}$ & 1 (dead) & 0 & 0 & 0 & NR \\
\hline Greenberg et al., 1979 & 2 & 65 & $2 \mathrm{M}$ & 0 & 0 & 0 & $\begin{array}{l}2 \text { (1 surv no disab, } \\
1 \text { dependent w/ } \\
\text { shunt) }\end{array}$ & 10 \\
\hline Feely, 1979 & 2 & 40.5 & $2 \mathrm{~F}$ & 0 & $\begin{array}{l}2 \text { (2 surv, } 1 \text { no disab, } \\
\quad 1 \mathrm{NR})\end{array}$ & 0 & 0 & NR \\
\hline $\begin{array}{l}\text { Schmidek \& Guthikon- } \\
\text { da, } 1979\end{array}$ & 1 & NR & NR & 0 & 1 (surv, outc NR) & 0 & 0 & NR \\
\hline Heros, 1979 & 1 & 22 & $1 \mathrm{M}$ & 0 & 0 & 1 (surv, outc NR) & 0 & NR \\
\hline Cuneo et al., 1979 & 1 & 23 & $1 \mathrm{M}$ & 1 (dead) & 0 & 0 & 0 & NR \\
\hline
\end{tabular}


Space-occupying cerebellar infarction

TABLE 3: Summary of available publications reporting on patients with space-occupying cerebellar infarction* (continued)

\begin{tabular}{|c|c|c|c|c|c|c|c|c|}
\hline Authors \& Year & $\begin{array}{l}\text { No. of } \\
\text { Pts } †\end{array}$ & $\begin{array}{c}\text { Mean } \\
\text { Age (yrs) }\end{array}$ & Sex & $\begin{array}{l}\text { Conservative } \\
\text { Treatment }\end{array}$ & SDC w/o EVD & SDC w/ EVD & EVD Alone & $\begin{array}{l}\text { Mean FU in } \\
\text { Mos (range) }\end{array}$ \\
\hline Spinnler et al., 1979 & 6 & NR & NR & $\begin{array}{l}1 \text { (surv, outc } \\
\text { NR) }\end{array}$ & \multicolumn{2}{|c|}{5 (2 dead, 3 surv, outc NR) } & 0 & NR \\
\hline Jones et al., 1980 & NR & NR & NR & NR & NR & NR & NR & NR \\
\hline Scotti et al., 1980 & 6 & NR & NR & $\begin{array}{l}1 \text { (surv, outc } \\
\text { NR) }\end{array}$ & $\begin{array}{l}5(5+)(2 \text { dead, } 3 \text { surv, } \\
\quad \text { outc NR) }\end{array}$ & 0 & 0 & NR \\
\hline Patrick et al., 1980 & 2 & NR & NR & NR & 2 (NR) & 0 & 0 & NR \\
\hline Woodhurst, 1980 & 4 & NR & NR & 0 & $\begin{array}{l}2 \text { (1 dead, } 1 \text { surv, outc } \\
\quad \text { NR) }\end{array}$ & $\begin{array}{l}2 \text { (1 dead, } 1 \text { surv, } \\
\quad \text { outc NR) }\end{array}$ & 0 & NR \\
\hline Hinshaw et al., 1980 & NR & NR & NR & NR & 0 & 0 & 0 & NR \\
\hline Sayama et al., 1981 & 5 & NR & NR & 0 & 4 (4 surv, outc NR) & 0 & 1 (dead) & NR \\
\hline Bergen et al., 1981 & 1 & 0.8 & $1 \mathrm{M}$ & 0 & 0 & 0 & $\begin{array}{l}1 \text { (surv w/ shunt, } \\
\text { outc "normal") }\end{array}$ & 30 \\
\hline Ho et al., 1981 & 3 & 71.3 & $1 \mathrm{~F}, 2 \mathrm{M}$ & $\begin{array}{l}2 \text { (2 surv, outc } \\
\quad \text { NR) }\end{array}$ & 1 (surv, outc NR) & 0 & 0 & NR \\
\hline $\begin{array}{l}\text { Shenkin \& Zavala, } \\
\quad 1982\end{array}$ & 6 & 61.8 & $2 \mathrm{~F}, 4 \mathrm{M}$ & 0 & 0 & 0 & $\begin{array}{l}6 \text { (6 surv, outc } 4 \\
\text { independent, } 2 \\
\text { mild-mod dis- } \\
\text { ab, } 4 \mathrm{w} / \text { shunt) }\end{array}$ & NR \\
\hline Taneda et al., 1982 & 15 & 59.3 & $2 \mathrm{~F}, 13 \mathrm{M}$ & 5 (5 dead) & $\begin{array}{l}7 \text { (1 dead, } 6 \text { surv, no } \\
\text { disab) }\end{array}$ & $\begin{array}{l}3 \text { (1 dead, } 2 \text { surv, } \\
1 \text { no disab, } 1 \\
\text { NR) }\end{array}$ & 0 & NR \\
\hline Harbaugh et al., 1982 & 1 & 13 & $1 \mathrm{M}$ & 0 & 0 & 0 & 1 (surv, outc NR) & 2 \\
\hline Khan et al., 1983 & 11 & 64.2 & $5 \mathrm{~F}, 6 \mathrm{M}$ & $\begin{array}{l}6 \text { (6 surv, } 2 \text { no } \\
\text { disab, } 4 \\
\text { mild-mod } \\
\text { disab) }\end{array}$ & 0 & 0 & $\begin{array}{l}5 \text { (1 dead, } 4 \text { mild- } \\
\text { mod disab) }\end{array}$ & NR \\
\hline $\begin{array}{l}\text { Horwitz \& Ludolph, } \\
1983\end{array}$ & 2 & 59 & $2 \mathrm{M}$ & $\begin{array}{c}1 \text { (surv, outc } \\
\text { NR) }\end{array}$ & 0 & 1 (surv, outc NR) & 0 & NR \\
\hline Shigemori et al., 1983 & 1 & 68 & $1 \mathrm{~F}$ & 0 & 0 & 0 & 1 (surv, mod disab) & 3 \\
\hline Derlon et al., 1983 & 1 & 19 & $1 \mathrm{M}$ & 0 & 0 & $\begin{array}{l}1(+)(\text { surv, outc } \\
\quad \text { NR) }\end{array}$ & 0 & NR \\
\hline $\begin{array}{l}\text { Duncan \& Forman, } \\
1984\end{array}$ & 1 & 75 & $1 \mathrm{M}$ & 0 & 0 & 0 & $\begin{array}{r}1 \text { (surv, mild-mod } \\
\text { disab w/ shunt) }\end{array}$ & 0.7 \\
\hline Laun et al., 1984 & 7 & 47.7 & $3 \mathrm{~F}, 4 \mathrm{M}$ & 1 (dead) & 1 (surv, outc NR) & $\begin{array}{l}3 \text { (3 surv, outc } \\
\quad \text { NR) }\end{array}$ & $\begin{array}{l}2 \text { (2 surv, outc } 1 \\
\text { severe disab, } \\
1 \mathrm{NR} \text { ) }\end{array}$ & 2.5 \\
\hline Busse et al., 1984 & 8 & 52.5 & $4 \mathrm{~F}, 4 \mathrm{M}$ & 2 (2 dead) & $1(+)($ surv, outc NR) & $\begin{array}{l}4(4+) \text { ( } 4 \text { surv, } \\
\text { outc NR) }\end{array}$ & 1 (surv, outc NR) & 1.2 \\
\hline Keidel et al. 1984 & 3 & 60 & $1 \mathrm{~F}, 2 \mathrm{M}$ & 1 (dead) & 0 & 0 & 2 (2 surv, outc NR) & 1.1 \\
\hline $\begin{array}{l}\text { Rousseaux et al., } \\
\quad 1985\end{array}$ & 1 & 63 & $1 \mathrm{~F}$ & $\begin{array}{l}1 \text { (surv, outc } \\
\text { NR) }\end{array}$ & 0 & 0 & 0 & NR \\
\hline $\begin{array}{l}\text { Tomaszek \& Rosner, } \\
1985\end{array}$ & 1 & 51 & $1 \mathrm{~F}$ & 1 (dead) & 0 & 0 & 0 & NR \\
\hline Cioffi et al., 1985 & 10 & 53.6 & $4 \mathrm{~F}, 6 \mathrm{M}$ & $\begin{array}{l}5 \text { (5 surv, outc } \\
\quad \text { NR) }\end{array}$ & 0 & 1 (surv, outc NR) & $\begin{array}{l}4 \text { (1 dead, } 3 \text { surv, } \\
\text { outc NR) }\end{array}$ & 24 \\
\hline Auer et al., 1986 & 13 & NR & NR & $\begin{array}{l}5 \text { (1 dead, } 4 \\
\quad \text { surv, outc } \\
\text { NR) }\end{array}$ & 7 (7 surv, outc NR) & 0 & 1 (dead) & NR \\
\hline
\end{tabular}

(continued) 


\section{H. Neugebauer et al.}

TABLE 3: Summary of available publications reporting on patients with space-occupying cerebellar infarction* (continued)

\begin{tabular}{|c|c|c|c|c|c|c|c|c|}
\hline Authors \& Year & $\begin{array}{l}\text { No. of } \\
\text { Pts† }\end{array}$ & $\begin{array}{c}\text { Mean } \\
\text { Age (yrs) }\end{array}$ & Sex & $\begin{array}{l}\text { Conservative } \\
\text { Treatment }\end{array}$ & SDC w/o EVD & SDC w/ EVD & EVD Alone & $\begin{array}{l}\text { Mean FU in } \\
\text { Mos (range) }\end{array}$ \\
\hline Macdonell et al., 1987 & 3 & NR & NR & 1 (dead) & $\begin{array}{l}2 \text { (1 dead, } 1 \text { surv, outc } \\
\text { NR) }\end{array}$ & 0 & 0 & NR \\
\hline Busse \& Laun, 1988 & 24 & NR & NR & $\begin{array}{l}5 \text { (3 dead, } 2 \\
\text { surv, outc } \\
\text { NR) }\end{array}$ & $\begin{array}{l}6(6+) \text { (1 dead, } 5 \text { surv, } \\
4 \text { mild-mod disab, } \\
1 \text { severe disab) }\end{array}$ & $\begin{array}{l}7 \text { (7 surv, } 6 \text { mild- } \\
\text { mod disab, } \\
1 \text { severe disab) }\end{array}$ & $\begin{array}{l}6 \text { (1 dead, } 5 \text { surv, } \\
3 \text { mild-mod } \\
\text { disab, } 2 \text { severe } \\
\text { disab) }\end{array}$ & 3 \\
\hline $\begin{array}{l}\text { Krieger \& Aschoff, } \\
1989\end{array}$ & 1 & 45 & $1 \mathrm{M}$ & 0 & 0 & $\begin{array}{l}1(+) \text { (surv, outc } \\
\quad \text { NR) }\end{array}$ & 0 & NR \\
\hline Hojer et al., 1990 & 9 & NR & NR & $\begin{array}{l}5 \text { (2 dead, } 3 \\
\text { surv, outc } \\
\text { NR) }\end{array}$ & $1(+)$ (surv, outc NR) & 0 & $\begin{array}{l}3 \text { (1 dead, } 2 \text { surv, } \\
\text { outc NR) }\end{array}$ & NR \\
\hline Andoh et al., 1990 & 9 & NR & NR & NR & NR & NR & $\begin{array}{l}9 \text { (surv, outcome } \\
\quad \text { NR) }\end{array}$ & NR \\
\hline $\begin{array}{l}\text { Klugkist \& McCarthy, } \\
1991\end{array}$ & 3 & 49.3 & $3 \mathrm{M}$ & 0 & 1 (surv, severe disab) & $\begin{array}{l}1 \text { (surv, mild-mod } \\
\text { disab) }\end{array}$ & $\begin{array}{l}1 \text { (surv, mild-mod } \\
\quad \text { disab) }\end{array}$ & $27.7(\max 54)$ \\
\hline Hojer et al., 1991 & 8 & NR & NR & 1 (dead) & 0 & 1 (surv, outc NR) & $\begin{array}{l}6 \text { (6 surv, outc } \\
\quad \text { NR) }\end{array}$ & NR \\
\hline Amarenco et al., 1991 & 6 & NR & NR & 6 (6 dead) & 0 & 0 & 0 & NR \\
\hline Struck et al., 1991 & 4 & NR & NR & 2 (2 dead) & 1 (surv, GOS 2) & 0 & 1 (surv, outc NR) & NR \\
\hline Chen et al., 1992 & 11 & 54.1 & $4 \mathrm{~F}, 7 \mathrm{M}$ & 0 & 0 & $\begin{array}{l}11 \text { (11 surv, } 6 \\
\text { mild-mod } \\
\text { disab, } 3 \text { severe } \\
\text { disab, } 2 \text { outc } \\
\text { NR) }\end{array}$ & 0 & $(16-60)$ \\
\hline Antonello et al., 1992 & 1 & 73 & $1 \mathrm{M}$ & 0 & 0 & 0 & 1 (surv, outc NR) & NR \\
\hline $\begin{array}{l}\text { Bertalanffy \& de } \\
\quad \text { Vries, } 1992\end{array}$ & 10 & 61.8 & $6 \mathrm{~F}, 4 \mathrm{M}$ & 0 & 0 & 0 & $\begin{array}{l}10 \text { (3 dead, } 6 \text { surv, } \\
\text { outc NR, } 1 \text { NR) }\end{array}$ & NR \\
\hline \multirow[t]{2}{*}{ Langmayr et al., 1993} & 15 & NR & NR & 0 & $8(8+)$ & $4(4+)$ & 3 & 3 \\
\hline & & & & & \multicolumn{2}{|c|}{$\begin{array}{l}\text { (outc I [no Sx]: 1; II [mild-mod disab]: 2; III } \\
\text { [severe disab]: 3; IV (dead): } 6\end{array}$} & (outc NR) & \\
\hline Kase et al., 1993 & 10 & NR & NR & 3 (3 dead) & $6(2+)(6$ outc NR) & 0 & 1 (outc NR) & NR \\
\hline Kase \& Wolf, 1993 & 1 & 55 & $1 \mathrm{M}$ & 0 & 0 & $\begin{array}{l}1 \text { (1 surv, no } \\
\quad \text { disab) }\end{array}$ & 0 & 2 \\
\hline $\begin{array}{l}\text { Tulyapronchote et al., } \\
1993\end{array}$ & 1 & 62 & $1 \mathrm{M}$ & 0 & 0 & 0 & 1 (surv mild disab) & NR \\
\hline Krieger et al., 1993 & 11 & 52 & $3 \mathrm{~F}, 8 \mathrm{M}$ & 0 & $\begin{array}{l}11(11+)(4 \text { dead, } 7 \text { surv, } \\
\quad \text { outc NR) }\end{array}$ & 0 & 0 & NR \\
\hline Tohgi et al., 1993 & 72 & NR & NR & NR & NR & NR & NR & 3 \\
\hline \multirow[t]{2}{*}{ Hornig et al., 1994} & 52 & 61.2 & $18 \mathrm{~F}$ & 16 & $8(8+)$ & 22 & 6 & NR \\
\hline & & & $34 \mathrm{M}$ & $\begin{array}{l}\text { (2 dead, } 14 \\
\text { surv, outc } \\
11 \text { no or } \\
\text { mild disab, } \\
3 \text { NR) }\end{array}$ & \multicolumn{2}{|c|}{$\begin{array}{l}\text { (4 dead, } 26 \text { surv, outc } 14 \text { mild-mod disab, } \\
12 \mathrm{NR} \text { ) }\end{array}$} & $\begin{array}{l}\text { (2 dead, } 4 \text { surv } \\
\text { mild-mod } \\
\text { disab) }\end{array}$ & \\
\hline Kanis et al., 1994 & 1 & 60 & $1 \mathrm{M}$ & 0 & 0 & 1 (dead) & 0 & 1 \\
\hline Miyachi et al., 1994 & 1 & 49 & $1 \mathrm{M}$ & 0 & 0 & 0 & 1 (dead) & 3 \\
\hline Tada et al., 1994 & 1 & 65 & $1 \mathrm{M}$ & $\begin{array}{l}1 \text { (surv, outc } \\
\text { NR) }\end{array}$ & 0 & 0 & 0 & 1.8 \\
\hline Ito et al., 1994 & 2 & 66.5 & $2 \mathrm{~F}$ & 0 & 0 & 0 & $\begin{array}{l}2 \text { (2 surv, outc } \\
\quad \text { NR) }\end{array}$ & NR \\
\hline Miyata et al., 1994 & 1 & 11 & $1 \mathrm{~F}$ & 0 & $1(1+)$ & 0 & 0 & 36 \\
\hline
\end{tabular}


Space-occupying cerebellar infarction

TABLE 3: Summary of available publications reporting on patients with space-occupying cerebellar infarction* (continued)

\begin{tabular}{|c|c|c|c|c|c|c|c|c|}
\hline Authors \& Year & $\begin{array}{l}\text { No. of } \\
\text { Pts† }\end{array}$ & $\begin{array}{c}\text { Mean } \\
\text { Age (yrs) }\end{array}$ & Sex & $\begin{array}{l}\text { Conservative } \\
\text { Treatment }\end{array}$ & SDC w/o EVD & SDC w/ EVD & EVD Alone & $\begin{array}{l}\text { Mean FU in } \\
\text { Mos (range) }\end{array}$ \\
\hline $\begin{array}{l}\text { Ogasawara et al., } \\
1995\end{array}$ & 10 & 59 & $3 F, 7 M$ & 0 & 0 & $\begin{array}{l}10 \text { ( } 2 \text { dead, } 8 \text { surv, } \\
\text { outc } 3 \text { no disab, } \\
3 \text { mild disab, } \\
1 \text { severe disab, } \\
1 \text { NR) }\end{array}$ & 0 & $(23-101)$ \\
\hline Mathew et al., 1995 & 19 & NR & NR & $\begin{array}{l}6 \text { (3 dead, } 3 \\
\text { surv, outc } \\
\text { NR) }\end{array}$ & $\begin{array}{l}2 \text { (1 dead, } 1 \text { surv outc } \\
\text { "good") }\end{array}$ & $\begin{array}{l}2 \text { (2 surv outc } \\
\text { "good") }\end{array}$ & $\begin{array}{c}9 \text { ( } 3 \text { dead, } 6 \text { surv } \\
\text { outc } 5 \text { "good," } \\
1 \text { mod disab) }\end{array}$ & NR \\
\hline Turgut et al., 1996 & 5 & NR & NR & $\begin{array}{r}3 \text { (1 dead, } 2 \\
\mathrm{mRS} \leq 3)\end{array}$ & 2 (1 dead, $1 \mathrm{mRS} \leq 3$ ) & 0 & 0 & NR \\
\hline $\begin{array}{l}\text { Matsumoto et al., } \\
1996\end{array}$ & 1 & 61 & $1 \mathrm{M}$ & 0 & 0 & 1 (surv, outc NR) & 0 & 12 \\
\hline Sagoh et al., 1997 & 1 & 38 & $1 \mathrm{M}$ & 0 & 0 & 0 & 1 (surv, outc NR) & 12 \\
\hline Calli et al., 1998 & 1 & 42 & $1 \mathrm{M}$ & 0 & 0 & 1 (surv, outc NR) & 0 & NR \\
\hline Wijdicks et al., 1998 & 1 & 47 & $1 \mathrm{M}$ & 0 & 0 & 0 & 1 (surv, outc NR) & NR \\
\hline $\begin{array}{l}\text { Mohsenipour et al., } \\
1999\end{array}$ & NR & $N R$ & NR & NR & NR & NR & NR & $\min 6$ \\
\hline Jauss et al., 1999 & 84 & 58.5 & $\begin{array}{l}28 \mathrm{~F} \\
56 \mathrm{M}\end{array}$ & $\begin{array}{l}36(30 \mathrm{mRS} \\
0-1)\end{array}$ & \multicolumn{2}{|c|}{34 (22 mRS 0-1, 12 outc NR) } & 14 (10 mRS 0-1) & 3 \\
\hline Skurnik et al., 1999 & NR & NR & NR & NR & NR & NR & NR & NR \\
\hline Koh et al., $2000^{63}$ & 35 & NR & NR & $\begin{array}{l}26 \text { (1 dead, } \\
25 \text { surv, } \\
\text { outc } 14 \\
\text { mRS } 0-2, \\
10 \text { mRS } \\
3-4,1 \mathrm{mRS} \\
5, \text { FU NR) }\end{array}$ & $\begin{array}{l}2 \text { (2 surv, outc } 1 \text { mRS } \\
3-4,1 \text { mRS } 5, \text { FU } \\
\text { NR) }\end{array}$ & $\begin{array}{l}1 \text { (surv, mRS 3- } \\
4, \text { FU NR) }\end{array}$ & $\begin{array}{l}6 \text { (1 dead, } 5 \text { surv, } \\
\text { outc } 2 \text { mRS 0- } \\
2,3 \text { mRS 3-4, } \\
\text { FU NR) }\end{array}$ & $16(1-105)$ \\
\hline Koh et al., $2000^{64}$ & 2 & 51.5 & $1 \mathrm{~F}, 1 \mathrm{M}$ & 0 & 0 & $\begin{array}{l}2 \text { (1 dead, } 1 \text { surv } \\
\text { GOS } 5)\end{array}$ & 0 & NR \\
\hline Hirayama et al., 2000 & 5 & NR & NR & 0 & 0 & $\begin{array}{l}1 \text { (surv, indepen- } \\
\text { dent) }\end{array}$ & 4 (4 NR) & 3 \\
\hline Raco et al., 2003 & 17 & NR & NR & NR & $\begin{array}{r}4(4+) \text { (2 dead, } 2 \text { surv } \\
1 \text { GOS 4, } 1 \text { GOS 3) }\end{array}$ & $\begin{array}{l}5(5+)(1 \text { dead, } 4 \\
\text { surv, } 3 \text { GOS } 5 \\
1 \text { GOS } 4)\end{array}$ & $\begin{array}{c}8 \text { (8 surv } 7 \text { GOS } \\
5,1 \text { GOS } 4)\end{array}$ & NR \\
\hline $\begin{array}{l}\text { Pérez Navero et al., } \\
2003\end{array}$ & 1 & 4 & $1 \mathrm{~F}$ & 1 (dead) & 0 & 0 & 0 & NR \\
\hline $\begin{array}{l}\text { Ganapathy et al., } \\
2003\end{array}$ & 1 & 61 & $1 \mathrm{~F}$ & 0 & 0 & $1(1+)(\mathrm{mRS} 3)$ & 0 & NR \\
\hline Prat et al., 2004 & 2 & 70 & $1 \mathrm{~F}, 1 \mathrm{M}$ & $\begin{array}{l}1 \text { (surv, outc } \\
\text { NR) }\end{array}$ & $1(+)($ surv, outc NR) & 0 & 0 & NR \\
\hline $\begin{array}{l}\text { Czernicki \& Marchel, } \\
2004\end{array}$ & 5 & NR & NR & NR & NR & NR & NR & NR \\
\hline Rupprecht et al., 2004 & 1 & 59 & $1 \mathrm{M}$ & 0 & 0 & 0 & 1 (surv, outc NR) & 1 \\
\hline $\begin{array}{l}\text { Jensen \& St. Louis, } \\
\quad 2005\end{array}$ & 1 & 48 & $1 \mathrm{~F}$ & $\begin{array}{l}1 \text { (surv, outc } \\
\quad N R \text { ) }\end{array}$ & 0 & 0 & 0 & NR \\
\hline Baldauf et al., 2006 & 10 & 62 & $4 \mathrm{~F}, 6 \mathrm{M}$ & 0 & 0 & 1 (surv, GOS 2) & $\begin{array}{l}9 \text { (9 surv, } 1 \text { GOS } 2 \\
4 \text { GOS } 3,2 \\
\text { GOS } 4,2 \text { GOS } \\
5)\end{array}$ & NR \\
\hline $\begin{array}{l}\text { Ramos-Zuñiga \& } \\
\text { Jiménez-Guerra, } \\
2006\end{array}$ & 8 & 63.5 & $3 F, 5 \mathrm{M}$ & $\begin{array}{l}3 \text { (3 surv, outc } \\
\text { NR) }\end{array}$ & 0 & 0 & $\begin{array}{l}5 \text { (5 surv, outc } \\
\quad \text { NR) }\end{array}$ & 3 \\
\hline
\end{tabular}


TABLE 3: Summary of available publications reporting on patients with space-occupying cerebellar infarction* (continued)

\begin{tabular}{|c|c|c|c|c|c|c|c|c|}
\hline Authors \& Year & $\begin{array}{l}\text { No. of } \\
\text { Pts† }\end{array}$ & $\begin{array}{c}\text { Mean } \\
\text { Age (yrs) }\end{array}$ & Sex & $\begin{array}{c}\text { Conservative } \\
\text { Treatment }\end{array}$ & SDC w/o EVD & SDC w/ EVD & EVD Alone & $\begin{array}{l}\text { Mean FU in } \\
\text { Mos (range) }\end{array}$ \\
\hline Rosi et al., 2006 & 12 & NR & NR & 1 (outc NR) & $\begin{array}{l}7 \text { ( } 2 \text { dead, } 5 \text { surv, outc } \\
\text { NR.) }\end{array}$ & 0 & $\begin{array}{l}4 \text { ( } 3 \text { dead, } 1 \text { surv, } \\
\text { outc NR) }\end{array}$ & NR \\
\hline Kudo et al., 2007 & 25 & 62.6 & $4 \mathrm{~F}, 21 \mathrm{M}$ & 0 & $\begin{array}{l}5(3+)(1 \text { dead, } 4 \text { surv } \\
1 \text { GOS } 5,2 \text { GOS } 4 \\
1 \text { GOS } 3)\end{array}$ & $\begin{array}{l}17(14+) \text { (1 dead, } \\
16 \text { surv, } 9 \text { GOS } \\
5,4 \text { GOS } 4,3 \\
\text { GOS } 3)\end{array}$ & $\begin{array}{l}3 \text { (1 dead, } 2 \text { surv, } \\
1 \text { GOS } 5,1 \\
\text { GOS } 3)\end{array}$ & NR \\
\hline Cerimagic et al., 2007 & 1 & 46 & $1 \mathrm{M}$ & 0 & 0 & 0 & 1 (surv, outc NR) & 4 \\
\hline Yoshimura et al., 2007 & 5 & 71.8 & $2 F, 3 \mathrm{M}$ & 0 & 0 & 0 & $\begin{array}{l}5 \text { (1 dead, } 4 \text { surv, } \\
2 \text { GOS } 5,1 \\
\text { GOS } 4,1 \text { GOS } \\
\text { 2) }\end{array}$ & 3 \\
\hline Hsieh et al., 2008 & 1 & 51 & $1 \mathrm{M}$ & 0 & 0 & 0 & 1 (surv, outc NR) & 1 \\
\hline Mangubat et al., 2009 & 4 & NR & NR & 0 & 0 & $\begin{array}{l}2 \text { (1 dead, } 1 \text { surv, } \\
\text { outc NR) }\end{array}$ & $\begin{array}{l}2 \text { (2 surv, outc } \\
\quad \text { NR) }\end{array}$ & NR \\
\hline Jüttler et al 2009 & $\begin{array}{c}56 \\
(25+)\end{array}$ & 57.7 & $\begin{array}{l}19 \mathrm{~F} \\
36 \mathrm{M}\end{array}$ & 0 & $\begin{array}{l}8 \text { (1 dead, } 7 \text { surv, } 2 \text { no } \\
\text { disab, } 5 \text { mild-mod } \\
\text { disab) }\end{array}$ & $\begin{array}{l}39 \text { (17 dead, } 22 \\
\text { surv, } 10 \text { no } \\
\text { disab, } 7 \text { mild- } \\
\text { mod disab, } 5 \\
\text { severe disab) }\end{array}$ & $\begin{array}{l}9 \text { (4 dead, } 5 \text { surv, } \\
4 \text { no disab, } 1 \\
\text { mild-mod } \\
\text { disab) }\end{array}$ & $96(41-143)$ \\
\hline $\begin{array}{l}\text { Pfefferkorn et al., } \\
2009\end{array}$ & 52 & 59.2 & $\begin{array}{l}18 \mathrm{~F} \\
34 \mathrm{M}\end{array}$ & 0 & $\begin{array}{l}52(52+) \text { (21 dead, } 31 \mathrm{su} \\
\text { mild-mod disab, } 4 \mathrm{se}\end{array}$ & $\begin{array}{l}\text { rv, } 13 \text { no disab, } 14 \\
\text { vere disab) }\end{array}$ & 0 & $55(12-132)$ \\
\hline Lungu et al., 2010 & 5 & NR & NR & 0 & $\begin{array}{l}5(5+) \text { (1 dead, } 4 \text { surv, } \\
\quad \text { outc NR) }\end{array}$ & 0 & 0 & 12 \\
\hline $\begin{array}{l}\text { Tsitsopoulos et al., } \\
2011114\end{array}$ & 10 & 54.9 & $2 \mathrm{~F}, 8 \mathrm{M}$ & 0 & 0 & $\begin{array}{l}10(10+) \text { ( } 2 \text { dead, } \\
8 \text { surv, } 1 \text { mRS0, } \\
2 \text { mRS1, } 3 \\
\text { MRS2, } 2 \text { mRS4) }\end{array}$ & 0 & $57.8(15-118)$ \\
\hline $\begin{array}{l}\text { Tsitsopoulos et al., } \\
2011115\end{array}$ & 32 & 64.3 & $8 \mathrm{~F}, 24 \mathrm{M}$ & 0 & 0 & $\begin{array}{l}32(32+) \text { (10 dead, } \\
22 \text { surv, } 17 \\
\text { mRS 0-2, } 5 \\
\text { mRS 3-5) }\end{array}$ & 0 & $67.5(14-113)$ \\
\hline $\begin{array}{l}\text { Montgomery et al., } \\
2012\end{array}$ & 4 & 8 & $4 \mathrm{M}$ & 0 & 0 & $\begin{array}{l}4(2+) \text { (2 surv, } 2 \\
\text { mild disab, } 2 \\
\text { mod disab) }\end{array}$ & 0 & $36(12-60)$ \\
\hline
\end{tabular}

* Shading indicates combined categories. Abbreviations: disab = disability; FU = follow-up; $\max =$ maximum; $\min =$ minimum; $\bmod =$ moderate; NR = not reported; outc = outcome; pts = patients; surv = survived; Sx = symptoms; + = strokectomy.

$\uparrow$ Number of patients with space-occupying cerebellar infarction.

3 studies on long-term outcome that include larger numbers of patients with follow-up of more than 1 year. ${ }^{56,91,115}$ In one of these studies, a retrospective single-center case series on SDC plus EVD and strokectomy (removal of necrotic tissue) in 32 patients with expansive unilateral cerebellar infarcts, a low mortality rate (32\%) and a high rate of independent outcome (53\% mRS score 0-2) was found in long-term survivors. ${ }^{115}$ In contrast, the 2 other studies, which included 108 patients treated with different strategies, reported a lower long-term survival rate of $60 \%$. Only $27 \%$ of survivors showed a favorable outcome (mRS score $0-1)$ and $38 \%$ showed an independent outcome (mRS score $0-2$ ). Age and the presence of brainstem infarction were identified as independent prognostic factors. Interestingly, insertion of an external ventricular drain, either alone or in combination with decompressive craniectomy, seemed to have no effect on survival or outcome..$^{56,91}$ These results call into question the belief that prognosis in survivors of space-occupying cerebellar infarcts is usually good. Indeed they indicate very heterogeneous outcome results, with $40 \%$ of patients dying and a considerable number of patients left with severe neurological deficits. However, the authors of these 2 studies were unable to assess the specific causes of death and severe disability in these patients. Both of the studies are retrospective and single center in nature and therefore also subject to bias.

\section{Suggested Treatment Algorithm}

The following treatment algorithm is based on treatment protocols from experienced and expert tertiary centers where large numbers of patients are treated every 
year for space-occupying cerebellar infarctions (Fig. 1). Nevertheless, it represents only a recommendation that can be adapted to local standards and conditions.

Alert and clinically stable patients should initially be treated conservatively and monitored closely using clinical scales and repeated neuroimaging. ${ }^{54,57,94}$ If feasible, these patients should be admitted to an ICU or a dedicated stroke unit where neurosurgical interventions can be performed 24 hours a day.

Somnolent or clinically critical patients should ideally be admitted to an ICU, although they may also be cared for in a dedicated stroke unit. In contrast to the stable patients described above, these critically ill patients should not, under any circumstances, be admitted to a general ward. If neuroimaging shows extensive cerebellar stroke in these patients (quantitatively assessed according to the numerical 3-item CT score suggested by Jauss and coworkers ${ }^{52}$ ), prophylactic SDC with or without EVD should be considered. Some authors believe that deterioration of consciousness is the strongest predictor for dangerous space occupation and should be a prime factor in the decision to intervene surgically. ${ }^{69}$ However, because clinical signs as well as neuroradiological parameters of deterioration are unspecific or may be detected too late, some authors recommend early or preventive neurosurgical therapy. ${ }^{12,69,78}$ They argue that the risk of EVD and SDC is comparatively small and patients undergoing preventive surgery tend to recover better than patients undergoing emergency treatment after deterioration. Others argue that SDC should not generally be recommended in patients with a level of consciousness better than coma because its efficacy is unproven and it may cause unnecessary complications. ${ }^{51}$ Similarly, prophylactic osmotherapy can be considered if there is radiological evidence of edema, but given the lack of evidence it is not recommended.

In patients whose condition deteriorates, neuroimaging should be performed immediately and frequently to determine the appropriate treatment strategy and, if possible, to rule out extensive brainstem infarction. If the fourth ventricle is blocked and hydrocephalus is present, EVD or SDC or both are recommended, with ventriculostomy to be definitely followed by SDC if the patient's condition does not improve or if it worsens. ${ }^{12,41,94} \mathrm{Al}$ though ventriculostomy alone is sometimes considered dangerous due to possible upward herniation, this risk seems rather small. $15,41,47,58,68,69,94,116$ Patients with signs of quadrigeminal cistern and/or brainstem compression should undergo immediate SDC combined with or followed by EVD if they have signs of hydrocephalus that do not resolve after SDC. ${ }^{12,41,94}$ Some authors regard the combined approach of ventriculostomy and decompressive surgery as the first choice for patients with occlusive hydrocephalus. ${ }^{15,27,44,54}$

In comatose patients, neuroimaging and/or brainstem auditory evoked potential (BAEP) and somatosensory evoked potential (SEP) studies should be considered to detect brainstem damage, and some reports have emphasized the potential usefulness of the latter modalities. ${ }^{65,66}$ In patients without brainstem infarction and normal find-

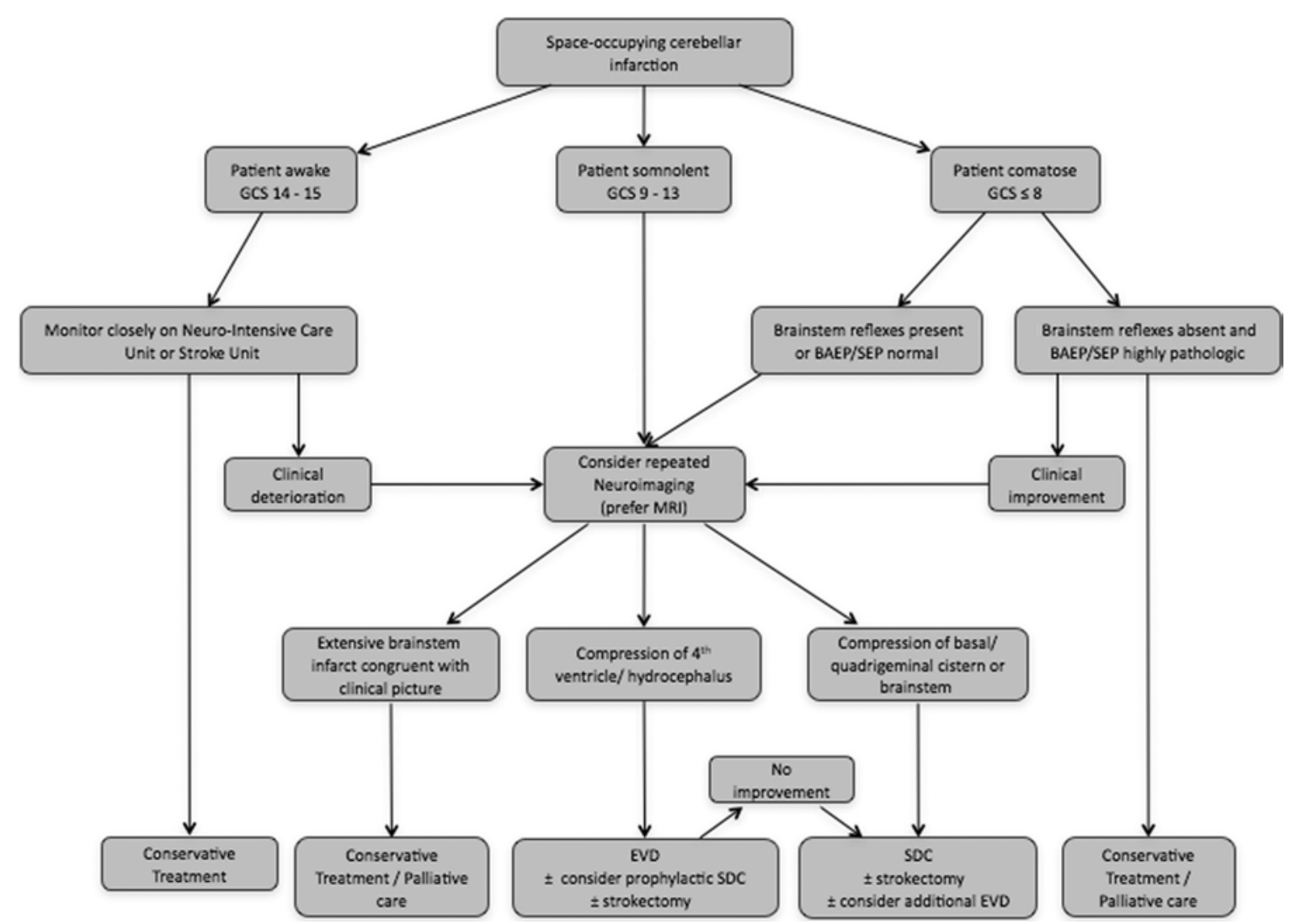

FIG 1. Cerebellar stroke treatment algorithm. Strokectomy refers to removal of necrotic tissue. GCS = Glasgow Coma Scale. 
ings on BAEP/SEP studies, immediate decompressive surgery plus EVD should be performed. For patients with extensive brainstem infarction or highly pathological BAEP/SEP findings, conservative treatment is recommended in view of the poor prognosis. If neither MRI nor BAEP/SEP studies can be performed, no general recommendation can be given, and therapeutic decisions have to be made on a case-by-case basis.

\section{Conclusions}

Although most cerebellar infarctions have a benign course, the early identification of those patients who are at risk for space-occupying edema formation is essential. In addition to monitoring patients closely and using MRI rather than CT for diagnostic evaluation, one should keep in mind that deterioration can occur late and must not be overlooked. Bilateral decompression craniotomy, including resection of the posterior arch of the atlas and in combination with dura enlargement, is thought to be the treatment of choice, even if patients present with a reduced level of consciousness. The available data, however, do not provide sufficient evidence to suggest the optimal timing of surgery or the optimal surgical procedure. Patients with extensive brainstem damage, however, have a poor outcome, and thus seem not to be good candidates for surgery. Furthermore, recent studies have shown that long-term outcome after space-occupying cerebellar infarction is more heterogeneous than expected, and therefore more studies are needed to identify prognostic factors. Randomized controlled trials would be needed to define the exact value of SDC, ventriculostomy, or a combination of both. Ethically, however, these trials are highly problematic because they might mean withholding from patients a potentially life-saving therapy such as SDC. It is the great challenge for neurosurgeons and neurologists to overcome these problems and create adequately designed trials that answer the open questions about space-occupying cerebellar infarction.

\section{Disclosure}

The authors report no conflict of interest concerning the materials or methods used in this study or the findings specified in this paper.

Author contributions to the study and manuscript preparation include the following. Conception and design: Neugebauer, Witsch, Jüttler. Acquisition of data: Neugebauer, Jüttler. Analysis and interpretation of data: Neugebauer, Zweckberger, Jüttler. Drafting the article: Neugebauer, Witsch, Jüttler. Critically revising the article: all authors. Reviewed submitted version of manuscript: all authors. Approved the final version of the manuscript on behalf of all authors: Neugebauer. Statistical analysis: Neugebauer, Jüttler. Administrative/technical/material support: Neugebauer, Jüttler. Study supervision: Neugebauer, Jüttler.

\section{References}

1. Adams HP Jr, del Zoppo G, Alberts MJ, Bhatt DL, Brass L, Furlan A, et al: Guidelines for the early management of adults with ischaemic stroke: a guideline from the American Heart Association/American Stroke Association Stroke Council, Clinical Cardiology Council, Cardiovascular Radiology and Intervention Council, and the Atherosclerotic Peripheral Vas- cular Disease and Quality of Care Outcomes in Research Interdisciplinary Working Groups: The American Academy of Neurology affirms the value of this guideline as an educational tool for neurologists. Circulation 115:e478-e534, 2007 (Erratum in Circulation 116:e515, 2007)

2. Amarenco P, Roullet E, Goujon C, Chéron F, Hauw JJ, Bousser MG: Infarction in the anterior rostral cerebellum (the territory of the lateral branch of the superior cerebellar artery). Neurology 41:253-258, 1991

3. Andoh T, Sakai N, Yamada H, Hattori T, Miwa Y, Hirata T, et al: [Cerebellar infarction: analysis of 33 cases.] No Shinkei Geka 18:821-828, 1990 (Jpn)

4. Antonello RM, Pasqua M, Bosco A, Torre P: Massive cerebellar infarct complicated by hydrocephalus. Ital J Neurol Sci 13:695-698, 1992

5. Arnow PM, Panwalker A, Garvin JS, Rodriguez-Erdmann F: Aspirin, hyperventilation, and cerebellar infarction in sickle cell disease. Arch Intern Med 138:148-149, 1978

6. Auer LM, Auer T, Sayama I: Indications for surgical treatment of cerebellar haemorrhage and infarction. Acta Neurochir (Wien) 79:74-79, 1986

7. Baldauf J, Oertel J, Gaab MR, Schroeder HW: Endoscopic third ventriculostomy for occlusive hydrocephalus caused by cerebellar infarction. Neurosurgery 59:539-544, 2006

8. Bardutzky J, Schwab S: Antiedema therapy in ischemic stroke. Stroke 38:3084-3094, 2007

9. Bergen BJ, Batnitzky S, Morantz RA, Price HI: Cerebellar infarction with associated acute hydrocephalus due to vertebral artery occlusion in a child. Neurosurgery 8:383-387, 1981

10. Bertalanffy H, de Vries J: Management of cerebellar infarction with associated occlusive hydrocephalus. Clin Neurol Neurosurg 94:19-23, 1992

11. Busse O, Laun A: Therapie des raumfordernden Kleinhirninfarktes. Akt Neurol 15:6-8, 1988

12. Busse O, Laun A, Agnoli AL: [Obstructive hydrocephalus in cerebellar infarcts.] Fortschr Neurol Psychiatr 52:164-171, 1984 (Ger)

13. Calli C, Katranci N, Guzelbag E, Alper H, Yunten N: Retroclival epidural hematoma secondary to decompressive craniectomy in cerebellar infarction: MR demonstration. J Neuroradiol 25:229-232, 1998

14. Cerimagic D, Glavic J, Lovrencic-Huzjan A, Demarin V: Occlusion of vertebral artery, cerebellar infarction and obstructive hydrocephalus following cervical spine manipulation. Eur Neurol 58:248-250, 2007

15. Chen HJ, Lee TC, Wei CP: Treatment of cerebellar infarction by decompressive suboccipital craniectomy. Stroke 23:957961,1992

16. Cioffi FA, Bernini FP, Punzo A, D'Avanzo R: Surgical management of acute cerebellar infarction. Acta Neurochir (Wien) 74:105-112, 1985

17. Cohadon F, Guérin A, Castel JP, Leifer C, Caillé M, Leman P: [Pseudo-tumorous softening of the cerebellum.] Bord Med 3:285-297, 1970 (Fr)

18. Corcos A, Gaches J, Le Beau J: [Pseudosurgical form of ischemic pontocerebellar edema.] Ann Med Interne (Paris) 122: 861-865, $1971(\mathrm{Fr})$

19. Cuneo RA, Caronna JJ, Pitts L, Townsend J, Winestock DP: Upward transtentorial herniation: seven cases and a literature review. Arch Neurol 36:618-623, 1979

20. Czernicki T, Marchel A: [Results of treatment of cerebellar infarctions.] Neurol Neurochir Pol 38:37-43, 2004 (Polish)

21. De Reuck J, Vander Eecken H: Cerebellar infarction and internal hydrocephalus. Acta Neurol Belg 78:129-140, 1978

22. Derlon JM, Charbonneau P, Théron J, Houtteville JP, Bazin C: [Edematous cerebellar infarction in the young subject.] Neurochirurgie 29:423-428, $1983(\mathrm{Fr})$

23. Duncan GW, Parker SW, Fisher CM: Acute cerebellar infarction in the PICA territory. Arch Neurol 32:364-368, 1975 
24. Duncan JS, Forman A: Hydrocephalus after cerebellar infarction. Br Med J (Clin Res Ed) 289:1301-1302, 1984

25. European Stroke Organisation (ESO) Executive Committee: Guidelines for management of ischaemic stroke and transient ischaemic attack 2008. Cerebrovasc Dis 25:457-507, 2008

26. Fairburn B, Oliver LC: Cerebellar softening; a surgical emergency. BMJ 1:1335-1336, 1956

27. Feely MP: Cerebellar infarction. Neurosurgery 4:7-11, 1979

28. Ferguson L, Sundaresan N, Richardson R, Marsan R: Acute hemorrhagic cerebellar infarction following angiography. Surg Neurol 5:176-178, 1976

29. Fischer EG, Strand RD, Gilles FH: Cerebellar necrosis simulating tumor in infancy. J Pediatr 81:98-100, 1972

30. Fisher CM, Karnes WE, Kubik CS: Lateral medullary infarction-the pattern of vascular occlusion. J Neuropathol Exp Neurol 20:323-379, 1961

31. Ford FR, Clark D: Thrombosis of the basilar artery with softenings in the cerebellum and brain stem due to manipulation of the neck; a report of two cases with one post-mortem examination, reasons are given to prove that damage to the vertebral arteries is responsible. Bull Johns Hopkins Hosp 98:37-42, 1956

32. Ganapathy K, Girija T, Rajaram R, Mahendran S: Surgical management of massive cerebellar infarction. J Clin Neurosci 10:362-364, 2003

33. George B, Cophignon J, George C, Lougnon J: [Surgical aspects of cerebellar infarctions based upon a series of 79 cases (author's transl).] Neurochirurgie 24:83-88, 1978 (Fr)

34. Géraud G, Guillaume J, Lagarrigue J, Lazorthes Y, Bès A, Géraud J: [Pseudo-tumoral softening of the cerebellum.] Rev Neurol (Paris) 134:183-195, 1978 (Fr)

35. Germain A, Morvan A: Ramollissement cérébelleaux pseudotumoral. Bull Soc Med Hosp Paris 35:501-524, 1936

36. Goodhart SP, Davison C: Syndrome of the posterior inferior and anterior inferior cerebellar arteries and their branches. Arch Neurol Psychiatry 35:501-524, 1936

37. Graham DI, Adams H: "Idiopathic" thrombosis in the vertebrobasilar arterial system in young men. BMJ 1:26-28, 1972

38. Greenberg J, Skubick D, Shenkin H: Acute hydrocephalus in cerebellar infarct and hemorrhage. Neurology 29:409-413, 1979

39. Harbaugh RE, Saunders RL, Reeves AG: Pediatric cerebellar infarction: case report and review of the literature. Neurosurgery 10:593-596, 1982

40. Heros RC: Cerebellar infarction resulting from traumatic occlusion of a vertebral artery. Case report. J Neurosurg 51: 111-113, 1979

41. Heros RC: Surgical treatment of cerebellar infarction. Stroke 23:937-938, 1992

42. Hinshaw DB Jr, Thompson JR, Hasso AN, Casselman ES: Infarctions of the brainstem and cerebellum: a correlation of computed tomography and angiography. Radiology 137:105112,1980

43. Hirayama A, Yamasaki S, Miyata M: Clinical use of lidocaine for control of stroke oedema in the posterior cranial fossa accompanied by acute hydrocephalus. Acta Neurochir Suppl 76:317-321, 2000

44. Ho SU, Kim KS, Berenberg RA, Ho HT: Cerebellar infarction: a clinical and CT study. Surg Neurol 16:350-352, 1981

45. Hojer C, Beil C, Neveling M, Szelies B, Haupt WF: [Diagnosis and prognosis of acute cerebellar infarcts. A retrospective study.] Nervenarzt 61:482-490, 1990 (Ger)

46. Hojer C, Bewermeyer H, Huber M, Neveling M, Engel-Riedel W, Haupt WF: Kleinhirninfarkte und Kleinhirnblutungen. Eine vergleichende, retrospective Studie an 78 Patienten. Akt Neurol 18:88-94, 1991

47. Hornig CR, Rust DS, Busse O, Jauss M, Laun A: Space-occupying cerebellar infarction. Clinical course and prognosis. Stroke 25:372-374, 1994
48. Horwitz NH, Ludolph C: Acute obstructive hydrocephalus caused by cerebellar infarction. Treatment alternatives. Surg Neurol 20:13-19, 1983

49. Hsieh CY, Lee JS, Yu CY, Chen CH: Bilateral cerebellar infarction in the medial branches of posterior inferior cerebellar arterial territory-using endoscopic third ventriculostomy to relieve acute hydrocephalus. Clin Neurol Neurosurg 110:7174, 2008

50. Ito M, Sonokawa T, Mishina H, Hishii M, Sato K: Surgical management of comatose patients with cerebellar infarction. J Clin Neurosci 1:251-256, 1994

51. Jauss M, Krieger D, Hornig C, Schramm J, Busse O: Surgical and medical management of patients with massive cerebellar infarctions: results of the German-Austrian Cerebellar Infarction Study. J Neurol 246:257-264, 1999

52. Jauss M, Müffelmann B, Krieger D, Zeumer H, Busse O: A computed tomography score for assessment of mass effect in space-occupying cerebellar infarction. J Neuroimaging 11:268-271, 2001

53. Jensen MB, St Louis EK: Management of acute cerebellar stroke. Arch Neurol 62:537-544, 2005

54. Jones HR Jr, Millikan CH, Sandok BA: Temporal profile (clinical course) of acute vertebrobasilar system cerebral infarction. Stroke 11:173-177, 1980

55. Jüttler E, Schellinger PD, Aschoff A, Zweckberger K, Unterberg A, Hacke W: Clinical review: therapy for refractory intracranial hypertension in ischaemic stroke. Crit Care 11:231, 2007

56. Jüttler E, Schweickert S, Ringleb PA, Huttner HB, Köhrmann M, Aschoff A: Long-term outcome after surgical treatment for space-occupying cerebellar infarction: experience in 56 patients. Stroke 40:3060-3066, 2009

57. Kanis KB, Ropper AH, Adelman LS: Homolateral hemiparesis as an early sign of cerebellar mass effect. Neurology 44:2194-2197, 1994

58. Kase CS, Norrving B, Levine SR, Babikian VL, Chodosh EH, Wolf PA, et al: Cerebellar infarction. Clinical and anatomic observations in 66 cases. Stroke 24:76-83, 1993

59. Kase CS, Wolf PA: Cerebellar infarction: upward transtentorial herniation after ventriculostomy. Stroke 24:1096-1098, 1993

60. Keidel M, Galle G, Wiedmayer J, Taghavy A: [Malignant cerebellar infarct.] Fortschr Neurol Psychiatr 52:277-283, 1984 (Ger)

61. Khan M, Polyzoidis KS, Adegbite AB, McQueen JD: Massive cerebellar infarction: "conservative" management. Stroke 14: 745-751, 1983

62. Klugkist H, McCarthy J: Surgical treatment of space-occupying cerebellar infarctions $-41 / 2$ years post-operative followup. Neurosurg Rev 14:17-22, 1991

63. Koh MG, Phan TG, Atkinson JL, Wijdicks EF: Neuroimaging in deteriorating patients with cerebellar infarcts and mass effect. Stroke 31:2062-2067, 2000

64. Koh MS, Goh KY, Tung MY, Chan C: Is decompressive craniectomy for acute cerebral infarction of any benefit? Surg Neurol 53:225-230, 2000

65. Krieger D, Adams HP, Rieke K, Hacke W: Monitoring therapeutic efficacy of decompressive craniotomy in space occupying cerebellar infarcts using brain-stem auditory evoked potentials. Electroencephalogr Clin Neurophysiol 88:261-270, 1993

66. Krieger D, Aschoff A: [Evoked potentials before and following decompression craniotomy in space-occupying cerebellar infarct.] Nervenarzt 60:36-39, 1989 (Ger)

67. Kubik CS, Adams RD: Occlusion of the basilar artery; a clinical and pathological study. Brain 69:73-121, 1946

68. Kudo H, Kawaguchi T, Minami H, Kuwamura K, Miyata M, Kohmura E: Controversy of surgical treatment for severe cerebellar infarction. J Stroke Cerebrovasc Dis 16:259-262, 2007 
69. Langmayr JJ, Buchberger W, Reindl H: [Cerebellar hemorrhage and cerebellar infarct: retrospective study of 125 cases.] Wien Med Wochenschr 143:131-133, 1993 (Ger)

70. Laun A, Busse O, Calatayud V, Klug N: Cerebellar infarcts in the area of the supply of the PICA and their surgical treatment. Acta Neurochir (Wien) 71:295-306, 1984

71. Lehrich JR, Winkler GF, Ojemann RG: Cerebellar infarction with brain stem compression. Diagnosis and surgical treatment. Arch Neurol 22:490-498, 1970

72. Levina G: [Cerebellar infarct in hypertensive crisis.] Arkh Patol 40:68-70, 1978 (Russian)

73. Lindgren SO: Infarctions simulating brain tumours in the posterior fossa. J Neurosurg 13:575-581, 1956

74. Lungu G, Mortada J, Stilhart B: [Surgical treatment of cerebellar infarction: five case studies.] Neurochirurgie 56:5962, 2010 (Ger)

75. Macdonell RA, Kalnins RM, Donnan GA: Cerebellar infarction: natural history, prognosis, and pathology. Stroke 18: 849-855, 1987

76. Mangubat EZ, Chan M, Ruland S, Roitberg BZ: Hydrocephalus in posterior fossa lesions: ventriculostomy and permanent shunt rates by diagnosis. Neurol Res 31:668-673, 2009

77. Masdeu JC, Irimia P, Asenbaum S, Bogousslavsky J, Brainin $\mathrm{M}$, Chabriat $\mathrm{H}$, et al: EFNS guideline on neuroimaging in acute stroke. Report of an EFNS task force. Eur J Neurol 13: 1271-1283, 2006

78. Mathew P, Teasdale G, Bannan A, Oluoch-Olunya D: Neurosurgical management of cerebellar haematoma and infarct. J Neurol Neurosurg Psychiatry 59:287-292, 1995

79. Matsumoto K, Kato A, Kohmura E, Fujinaka T, Fukuhara R: Acute cerebellar edema following intra-arterial tissue plasminogen activator thrombolysis for basilar artery occlusioncase report. Neurol Med Chir (Tokyo) 36:455-457, 1996

80. Menzies WF: Thrombosis of the inferior cerebellar artery. Brain 16:436-439, 1893

81. Miki K, Kurimoto T, Tani S, Ikeda Y, Kawamura Y, Someda $\mathrm{K}$, et al: [On the surgical treatment of cerebellar infarction (author's transl).] No Shinkei Geka 6:707-713, 1978 (Jpn)

82. Miyachi S, Okamura K, Watanabe M, Inoue N, Nagatani T, Takagi T: Cerebellar stroke due to vertebral artery occlusion after cervical spine trauma. Two case reports. Spine (Phila Pa 1976) 19:83-88, 1994

83. Miyata I, Imaoka T, Masaoka T, Nishiura T, Ishimitsu H: Pediatric cerebellar infarction caused by atlantoaxial subluxationcase report. Neurol Med Chir (Tokyo) 34:241-245, 1994

84. Mohsenipour I, Gabl M, Schutzhard E, Twerdy K: Suboccipital decompressive surgery in cerebellar infarction. Zentralbl Neurochir 60:68-73, 1999

85. Momose KJ, Lehrich JR: Acute cerebellar infarction presenting as a posterior fossa mass. Radiology 109:343-352, 1973

86. Montgomery AK, Maixner WJ, Wallace D, Wray A, Mackay MT: Decompressive craniectomy in childhood posterior circulation stroke: a case series and review of the literature. Pediatr Neurol 47:193-197, 2012

87. Norris JW, Eisen AA, Branch CL: Problems in cerebellar hemorrhage and infarction. Neurology 19:1043-1050, 1969

88. Ogasawara K, Koshu K, Nagamine Y, Fujiwara S, Mizoi K, Yoshimoto T: [Surgical decompression for massive cerebellar infarction.] No Shinkei Geka 23:43-48, 1995 (Jpn)

89. Patrick BK, Ramirez-Lassepas M, Synder BD: Temporal profile of vertebrobasilar territory infarction. Prognostic implications. Stroke 11:643-648, 1980

90. Pérez Navero JL, Montero-Shiemann C, Velasco Jabalquinto MJ, Ibarra de la Rosa I, Antón Gamero M, Salas Molina J: [Fatal outcome after severe cerebellar infarction due to spontaneous dissection of the vertebral artery.] An Pediatr (Barc) 58:385-389, 2003 (Span)

91. Pfefferkorn T, Eppinger U, Linn J, Birnbaum T, Herzog J, Straube A, et al: Long-term outcome after suboccipital decompressive craniectomy for malignant cerebellar infarction. Stroke 40:3045-3050, 2009
92. Pomme B, Terrasse J, Janny P, Gras H, Gibert J: [Pseudotumoral softening of the cerebellum; two cases.] Sem Hop 35:791793, 1959 (Fr)

93. Prat R, Conde FJ, Febles P, Cortés S, Millán-Corada AM: [Space-occupying cerebellar infarcts: surgical or conservative treatment?] Rev Neurol 38:133-136, 2004 (Span)

94. Raco A, Caroli E, Isidori A, Salvati M: Management of acute cerebellar infarction: one institution's experience. Neurosurgery 53:1061-1066, 2003

95. Ramos-Zuñiga R, Jiménez-Guerra R: Rational management of transient obstructive hydrocephalus secondary to a cerebellar infarct. Minim Invasive Neurosurg 49:302-304, 2006

96. Rosi J Jr, de Oliveira PG, Montanaro AC, Gomes S, Godoy R: [Cerebellar infarction: analysis of 151 patients.] Arq Neuropsiquiatr 64 (2B):456-460, 2006 (Portugese)

97. Rousseaux M, Devos P, Lesoin F, Petit H: "Pseudotumoral" cystic cerebellar infarction with slow evolution. Neurosurgery 16:61-63, 1985

98. Rupprecht TA, Weil S, Winkler PA, Kreuzer E, Pfister HW: Successful cardic surgery 24 hours after craniotomy in a patient with infective endocarditis and embolic cerebellar infarction: case report. J Heart Valve Dis 13:228-230, 2004

99. Sagoh M, Hirose Y, Murakami H, Katayama M, Akaji K, Mayanagi K: Cerebellar infarction with hydrocephalus caused by spontaneous extracranial vertebral artery dissection-case report. Neurol Med Chir (Tokyo) 37:538-541, 1997

100. Savitz MH, Katz SS, Nakazawa H: Acute cerebellar infarction producing brainstem compression. Mt Sinai J Med 44: 299-303, 1977

101. Sayama I, Sakotani AY, Yasui N, Ito Z, Kobayashi T, Nakajima K: [Cerebellar infarction-early prediction to the operative indication of posterior fossa decompression (author's transl).] No To Shinkei 33:801-810, 1981 (Jpn)

102. Schmidek HH, Guthikonda M: Acute cerebellar infarction-a potential surgical emergency, in Smith JL (ed): Neuroophthalmology Focus. New York: Masson Press, 1979, pp 427-432

103. Scotti G, Spinnler H, Sterzi R, Vallar G: Cerebellar softening. Ann Neurol 8:133-140, 1980

104. Shenkin HA, Zavala M: Cerebellar strokes: mortality, surgical indications, and results of ventricular drainage. Lancet 2: 429-432, 1982

105. Shigemori M, Ogata T, Tokutomi T, Yuge T, Watanabe M, Kuramoto S: Massive cerebellar infarction with obstructive hydrocephalus-a case report. Kurume Med J 30:81-84, 1983

106. Skurnik Y, Brandiner S, Gandelman G, Shtoeger Z: [Cerebellar infarction, clinical presentation, diagnosis and treatment.] Harefuah 136: 206-210, 1999 (Hebrew)

107. Spinnler H, Sterzi R, Vallar G: [Natural history, treatment and outcome of cerebellar softening (author's transl).] Riv Patol Nerv Ment 100:309-328, 1979 (Italian)

108. Struck LK, Biller J, Bruno A, Neiman RF, Yuh WTC, Marsh EE III, et al: Superior cerebellar artery territory infarction. Cerebrovasc Dis 1:71-75, 1991

109. Sypert GW, Alvord EC Jr: Cerebellar infarction. A clinicopathological study. Arch Neurol 32:357-363, 1975

110. Tada Y, Mizutani T, Nishimura T, Tamura M, Mori N: Acute bilateral cerebellar infarction in the territory of the medial branches of posterior inferior cerebellar arteries. Stroke 25:686-688, 1994

111. Taneda M, Ozaki K, Wakayama A, Yagi K, Kaneda H, Irino T: Cerebellar infarction with obstructive hydrocephalus. J Neurosurg 57:83-91, 1982

112. Tohgi H, Takahashi S, Chiba K, Hirata Y: Cerebellar infarction. Clinical and neuroimaging analysis in 293 patients. Stroke 24:1697-1701, 1993

113. Tomaszek DE, Rosner MJ: Cerebellar infarction: analysis of twenty-one cases. Surg Neurol 24:223-226, 1985 
114. Tsitsopoulos PP, Tobieson L, Enblad P, Marklund N: Clinical outcome following surgical treatment for bilateral cerebellar infarction. Acta Neurol Scand 123:345-351, 2011

115. Tsitsopoulos PP, Tobieson L, Enblad P, Marklund N: Surgical treatment of patients with unilateral cerebellar infarcts: clinical outcome and prognostic factors. Acta Neurochir (Wien) 153:2075-2083, 2011

116. Tulyapronchote R, Malkoff MD, Selhorst JB, Gomez CR: Treatment of cerebellar infarction by decompression suboccipital craniectomy. Stroke 24:478-480, 1993

117. Turgut M, Ozcan OE, Ertürk O, Saribaş O, Erbengi A: Spontaneous cerebellar strokes. Clinical observations in 60 patients. Angiology 47:841-848, 1996

118. Unterberg A, Juettler E: The role of surgery in ischemic stroke: decompressive surgery. Curr Opin Crit Care 13:175-179, 2007

119. Vereshchagin NV, Chebotareva NM, Kalashnikova LA: [Cerebellar infarct: clinical picture, diagnosis and treatment.] $\mathbf{Z h}$ Nevropatol Psikhiatr Im S S Korsakova 78:1609-1614, 1978 (Russian)

120. Wijdicks EFM, Maus TP, Piepgras DG: Cerebellar swelling and massive brain stem distortion: spontaneous resolu- tion documented by MRI. J Neurol Neurosurg Psychiatry 65:400-401, 1998

121. Wood MW, Murphey F: Obstructive hydrocephalus due to infarction of a cerebellar hemisphere. J Neurosurg 30:260 263,1969

122. Woodhurst WB: Cerebellar infarction - review of recent experiences. Can J Neurol Sci 7:97-99, 1980

123. Yoshimura K, Kubo S, Nagashima M, Hasegawa H, Thminaga S, Yoshimine T: Occlusive hydrocephalus associated with cerebellar infarction treated with endoscopic third ventriculostomy: report of 5 cases. Minim Invasive Neurosurg 50:270-272, 2007

Manuscript submitted October 25, 2012.

Accepted February 20, 2013.

Please include this information when citing this paper: DOI: 10.3171/2013.2.FOCUS12363.

Address correspondence to: Hermann Neugebauer, M.D., Rehabilitation and University Hospital Ulm, Department of Neurology, Oberer Eselsberg 45, 89081 Ulm, Germany. email: hermann. neugebauer@charite.de. 